\title{
The Ratio of Gluon Distributions in Sn and C*
}

\author{
T. Gousset and H.J. Pirner \\ Institut für Theoretische Physik, Universitåt Heidelberg \\ Philosophenweg 19, D-69120 Heidelberg, Germany
}

\begin{abstract}
We calculate the ratio of gluon densities, $G^{\mathrm{Sn}}(x) / G^{\mathrm{C}}(x)$, for $0.01<x<0.1$, from the new high statistics data on $F_{2}^{\mathrm{Sn}} / F_{2}^{\mathrm{C}}$ taken by the NM Collaboration. For small $x$, the shadowing in the gluon distribution is about equal to the shadowing of quark distribution. The antishadowing in the gluon distribution, however, is roughly $10 \%$. We also compare with the ratio difference $R=\sigma_{L} / \sigma_{T}$ from Sn and C.
\end{abstract}

HD-TVP-96-1

* Supported by the Federal Ministry of Education, Science, Research and Technology (BMBF) under grant no. 06 HD 742 
The investigation of nuclei with deep inelastic lepton scattering has produced very accurate data on nuclear structure functions $F_{2}^{\mathrm{A}}(x)$. Especially the measurement of the ratio of tin to carbon structure functions has been carried out recently with very high statistics [1]. It shows shadowing for $x \leq 0.05$. Antishadowing $(\leq 2 \%)$ is visible around $x=0.1$, i.e. at distance scales of $r=1 / x M_{N} \approx 2 \mathrm{fm}$ corresponding to the average distance between nucleons. In the interval $0.15 \leq x \leq 0.8$ the nuclear valence quark density is reduced. By measuring three different $\mu$-beam energies $(120,200$ and $280 \mathrm{GeV}) \mathrm{NMC}$ has also been able to determine the weak $\ln Q^{2}$ dependence of the structure function ratio. The aim of this note is to use the leading order evolution equations to estimate the little known underlying gluon densities.

There are older data [2] from inelastic $J / \psi$ production at $z \leq 0.85, p_{\perp}^{2} \geq 0.4(\mathrm{GeV} / c)^{2}$, which determine $G^{\mathrm{Sn}}(x) / G^{\mathrm{C}}(x) \approx 1.13 \pm 0.08$ in the interval $0.05 \leq x \leq 0.15$. Hadronic production of $J / \psi, \psi^{\prime}$ and $\Upsilon$ in $p A$ or $\pi A$ collisions [3] has been analysed with the gluon-gluon fusion process to get $G^{\mathrm{A}}\left(x_{2}\right) / G^{p}\left(x_{2}\right)$ in the region where the nuclear gluon momentum fraction $x_{2}$ is small [⿴囗十, 5]. In the small $x_{2}$ region, however, the second gluon is as soft as the third gluon which is necessary to form the $J / \psi^{3} S_{1}$-state. Therefore one expects final state interaction effects in the small $x_{2}$ region [6].

Currently there is a discussion to use nuclear beams in HERA. The study of the gluon distribution in nuclei at small $x$ would be one of the outstanding new opportunities to investigate nuclei on the parton level. Also the forthcoming heavy ion experiments at RHIC and LHC need a good understanding of the nuclear parton distributions to calculate the cross sections for hard processes initiated by nuclear collisions.

Measurements of the gluon distribution in nuclei give experimental windows viewing the partonic structure of nuclear binding. Very little is known about the role gauge fields play in the nucleus. This has been studied for the case of abelian QED in a model where the hydrogene atom replaces the nucleon. In this model, one computes the photon density of the hydrogene molecule, $G^{\mathrm{H}_{2}}(x)$, and compares it to the free atom photon density, $G^{\mathrm{H}}(x)$. The electron orbits of the hydrogene atoms in the molecule are polarized and modified by the electron exchange interaction leading to a suppression of photons at small $x$. At the momentum corresponding to the relative distance of the two protons a small antishadowing peak is visible [7]. In analogy, gluon antishadowing may indicate the relative distance $\left(x M_{N}\right)^{-1} \approx 2 \mathrm{fm}$ between the centers of nucleons, which act as a source for color fields. A covalent binding of quarks may manifest itself as a density dependent lack of long range gluons at $x<0.1$ similarly to the deformation of the photon cloud in the hydrogene molecule. In addition, in non-abelian QCD one expects at small $x$ processes where gluons from different nucleons overlap and merge [8]. Both effects have also an interpretation in the nuclear rest frame in terms of absorption of various hadronic components of the photon in the nucleus.

The strong theoretical interest, the excellent new data of NMC and the starting discussion of HERA with nuclei motivated us to extract from the available data the maximum information on the gluon distributions in nuclei. 
As usual, we define the Bjorken variable relative to nucleon kinematics

$$
x=\frac{Q^{2}}{2 M_{N} \nu},
$$

with $M_{N}$ the nucleon mass, $\nu$ the virtual photon energy in the laboratory and $Q^{2}=-q^{2}$ its virtuality. The Leading-Log (LL) approximation reads (for a review see Ref. [9])

$$
\begin{aligned}
F_{2}\left(x, Q^{2}\right) & =x \sum_{i} e_{i}^{2} q_{i}\left(x, Q^{2}\right), \\
\frac{\partial F_{2}}{\partial \ln Q^{2}} & =\frac{\alpha_{S}}{2 \pi} x\left(\sum_{i} e_{i}^{2} P_{q G} * G+P_{q q} * \frac{F_{2}}{x}\right),
\end{aligned}
$$

where $q_{i}$ and $G$ are the quark and gluon densities in the nucleon or in the nucleus (in the nuclear case, we shall consider distributions per nucleon). The splitting functions are denoted by $P_{q q}$ and $P_{q G}$. The sum runs over active flavors of quarks and antiquarks and $e_{i}$ is the corresponding charge. It is understood that distributions, structure functions, as well as $\alpha_{S}$ are $Q^{2}$ dependent. As we will see, for $x$ sufficiently small, the second term in the evolution equation of $F_{2}$ is small in comparison with the first. Furthermore, the first term can be approximated in a simple way to easily extract the gluon distribution from the evolution of $F_{2}$ [10. In the nucleon case, the LL approximation becomes insufficient at very small $x$. Next-to-LL (NLL) corrections are about $10-20 \%$ at $x=0.01$. For our purpose of extracting ratios in the range $10^{-2}-10^{-1}$, however, the LL approximation is accurate enough. In the nuclear case, additionnal merging terms are expected to play an important role in the evolution equation with the increase of the gluon density. These non-linear effects are expected to appear at very small $x\left(<10^{-2}\right)$ [8].

Let us consider the first term in Eq. (2). It can be written in detail as

$$
x \sum_{i} e_{i}^{2}\left(P_{q G} * G\right)=\frac{x}{2} \sum_{i} e_{i}^{2} \int_{x / A}^{1} \frac{d y}{y}\left(y^{2}+(1-y)^{2}\right) G(x / y) .
$$

We can replace the lower bound of the integral, $x / A$, by $x / A \rightarrow 0$, if in addition we impose $G(u) \equiv 0$ in the unphysical region, $u \geq A$. Because the gluon density is completly suppressed for $x \gtrsim 0.5$ this replacement is an accurate approximation in the small $x$ region for any reasonable ansatz for $G(x)$. We then expand

$$
g\left(\frac{x}{y}\right) \equiv \frac{x}{y} G\left(\frac{x}{y}\right)
$$

around $y=1 / 2$,

$$
g\left(\frac{x}{y}\right)=g(2 x)-4 x g^{\prime}(2 x)\left(y-\frac{1}{2}\right)+\left[(4 x)^{2} g^{\prime \prime}(2 x)+16 x g^{\prime}(2 x)\right] \frac{\left(y-\frac{1}{2}\right)^{2}}{2 !}+\cdots
$$

Carrying out the integration, odd terms in the expansion do not contribute and one gets

$$
\int_{0}^{1} d y\left(y^{2}+(1-y)^{2}\right) g(x / y)=\frac{2}{3} g(2 x)+\frac{2}{15}\left[(2 x)^{2} g^{\prime \prime}(2 x)+2(2 x) g^{\prime}(2 x)\right]+\cdots
$$




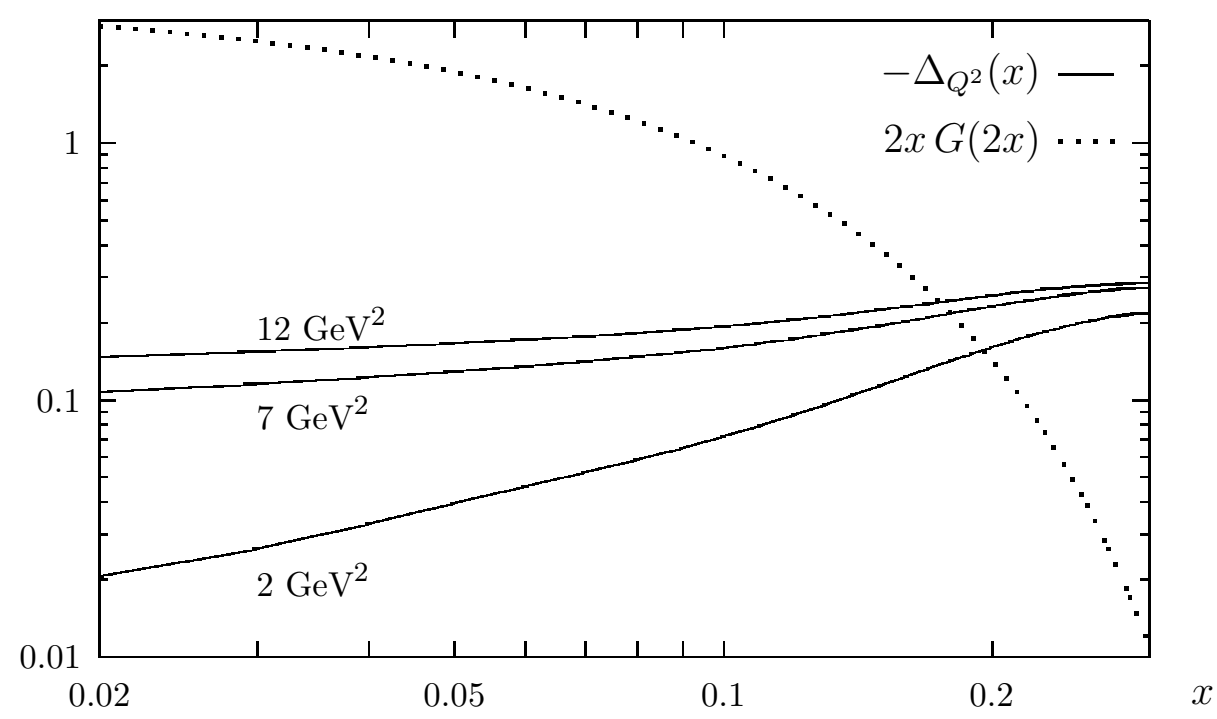

Figure 1: The correction $-\Delta(x)$ for different $Q^{2}$ in the nucleon case. The NMC fit to the gluon density [11], evaluated at $2 x$, is also plotted for comparison (the $Q^{2}$ for this density is $7 \mathrm{GeV}^{2}$ ).

As found in [10], this expansion is efficient for standard gluon distributions and one can approximate

$$
\int_{0}^{1} d y\left(y^{2}+(1-y)^{2}\right) g(x / y) \approx \frac{2}{3} g(2 x) .
$$

One can effectively check that for $g(u)=K u^{-\Delta}$, the right hand side of Eq. (雨) becomes

$$
\frac{2}{3} K(2 x)^{-\Delta}\left(1+\frac{\Delta(\Delta-1)}{5}+\cdots\right)
$$

showing that Eq. (5) is accurate for $0<\Delta<1$, with an error smaller than $5 \%$.

We now consider the second term in Eq. (2)

$$
\Delta\left(x, Q^{2}\right)=x\left(P_{q q} * \frac{F_{2}}{x}\right)=\frac{4}{3} \int_{x / A}^{1} d y\left(\frac{\left(1+y^{2}\right) F_{2}(x / y)-2 F_{2}(x)}{1-y}+\frac{3}{2} \delta(1-y) F_{2}(x / y)\right)
$$

One can compute $\Delta\left(x, Q^{2}\right)$ from $F_{2}$ data. We show in Fig. 1 the behavior of $\Delta$ as a function of $x$ and $Q^{2}$ in the case of the nucleon. The behavior of the gluon distribution is also plotted for comparison. For $x \approx 0.2, \Delta(x)$ is of the same order of magnitude as $2 x G(2 x)$ and indeed cancels the gluon contribution in Eq. (2) leading to the true scaling of $F_{2}$. Due to the steep rise of $G, \Delta$ becomes a small correction as soon as $x \leq 0.1$, thus implying a simple relation between $F_{2}$ evolution and gluon density.

In the following, we shall take into account both contributions to the evolution equation, Eq. (2), i.e. 


$$
\frac{\partial F_{2}}{\partial \ln Q^{2}} \approx \frac{\alpha_{S}}{2 \pi}\left(\Delta\left(x, Q^{2}\right)+\frac{2}{3} \frac{\sum_{i} e_{i}^{2}}{2} 2 x G\left(2 x, Q^{2}\right)\right) .
$$

We can now turn to the study of the new NMC data on tin to carbon ratio of structure functions. Namely, measurements of

$$
\begin{aligned}
f_{1}(x) & =\frac{F_{2}^{\mathrm{Sn}}}{F_{2}^{\mathrm{C}}} \\
f_{2}(x) & =\frac{\partial}{\partial \ln Q^{2}} \frac{F_{2}^{\mathrm{Sn}}}{F_{2}^{\mathrm{C}}},
\end{aligned}
$$

have been performed [1]. To be more precise, let us mention that the ratio, $f_{1}$, has been measured for fixed $x$ over a certain range of $Q^{2}$, so that the reported value is an average of ratios corresponding to an averaged $\left\langle Q^{2}\right\rangle$, where $\left\langle Q^{2}\right\rangle$ varies from one $x$ to the next. The evolution of ratio with $\ln Q^{2}$ has then been computed, giving the "slope", $f_{2}$, at $x$ and $\left\langle Q^{2}\right\rangle$.

Using Eq. (7), it is easy to relate the tin to carbon ratio of gluon distributions

$$
r\left(x, Q^{2}\right)=\left.\frac{G^{\mathrm{Sn}}}{G^{\mathrm{C}}}\right|_{x, Q^{2}},
$$

to $f_{1}$ and $f_{2}$, and one finds

$$
r\left(2 x,\left\langle Q^{2}\right\rangle\right)=f_{1}+\left.\frac{f_{2}-\frac{\alpha_{S}}{2 \pi} \frac{\Delta^{\mathrm{Sn}}-f_{1} \Delta^{\mathrm{C}}}{F_{2}^{\mathrm{C}}}}{f_{3}-\frac{\alpha_{S}}{2 \pi} \frac{\Delta^{\mathrm{C}}}{F_{2}^{\mathrm{C}}}}\right|_{x,\left\langle Q^{2}\right\rangle},
$$

where

$$
f_{3}=\frac{\partial \ln F_{2}^{\mathrm{C}}}{\partial \ln Q^{2}}=\frac{\partial \ln F_{2}^{d}}{\partial \ln Q^{2}}+\frac{\partial}{\partial \ln Q^{2}} \ln \frac{F_{2}^{\mathrm{C}}}{F_{2}^{d}} .
$$

In this analysis, we use the fact that the ratio of carbon to deuteron structure functions shows practically no $Q^{2}$ dependence [12], so that we can drop the second term in the right hand side of Eq. (11]), and use for $f_{3}$ the deuteron data of [11].

The extraction corresponding to Eq. (10) is given in Table 1 for each $x$ together with the corresponding $\left\langle Q^{2}\right\rangle$. We plot the ratio $r(x)$ in Fig. 2 together with $f_{1}(x)$. One

\begin{tabular}{ccccccccccc}
\hline \hline$x$ & 0.011 & 0.017 & 0.025 & 0.035 & 0.05 & 0.07 & 0.09 & 0.11 & 0.14 & 0.18 \\
$\left\langle Q^{2}\right\rangle$ & 1.8 & 2.4 & 3.4 & 4.4 & 5.7 & 7.3 & 8.6 & 9.6 & 11.2 & 12.8 \\
$r$ & 0.87 & 0.88 & 0.98 & 1.03 & 1.06 & 1.08 & 1.09 & 1.07 & 1.09 & 1.05 \\
$\delta r$ & 0.05 & 0.04 & 0.03 & 0.04 & 0.03 & 0.03 & 0.04 & 0.05 & 0.04 & 0.06 \\
\hline \hline
\end{tabular}

Table 1: The ratio of tin to carbon gluon density for different $x$ and corresponding $\left\langle Q^{2}\right\rangle . \delta r$ is the uncertainty on $r$ coming from the statistical error on the slope $f_{2}$. 


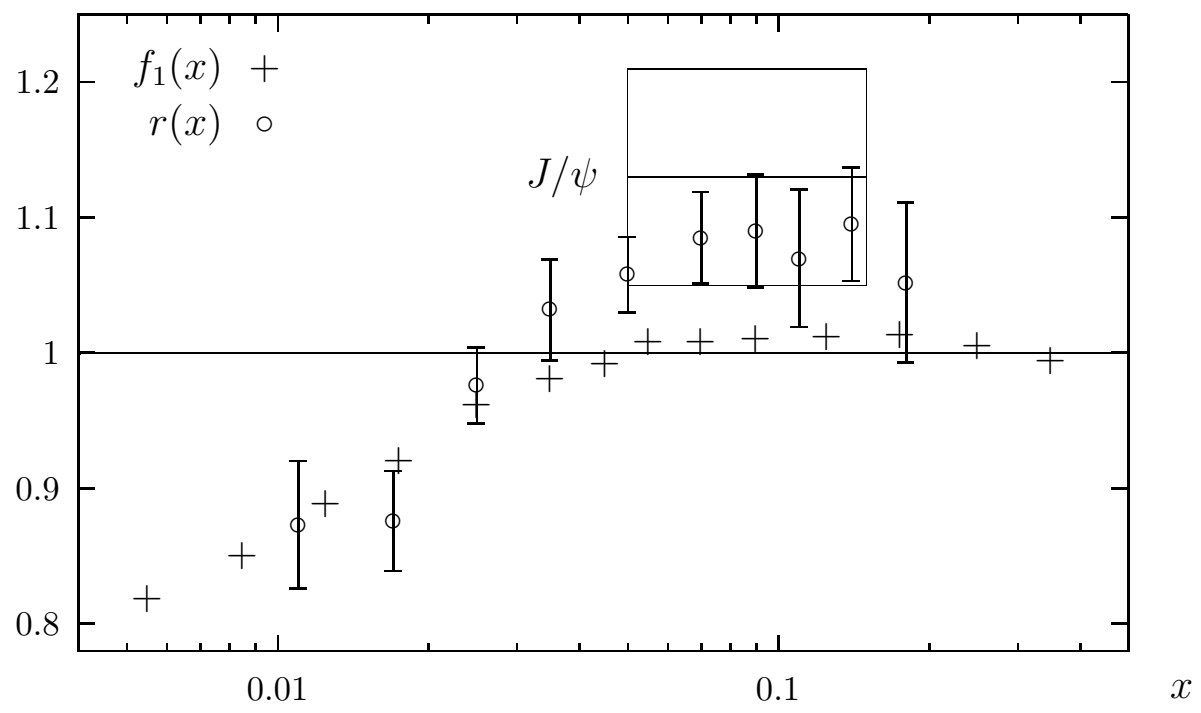

Figure 2: The ratio $r(x)=G^{\mathrm{Sn}}(x) / G^{\mathrm{C}}(x)$ of tin to carbon gluon density as a function of $x$ together with the ratio of structure function, $f_{1}(x)=F_{2}^{\mathrm{Sn}}(x) / F_{2}^{\mathrm{C}}(x)$. The statistical error on $f_{1}$ is less than $1 \%$ in the whole range of $x$. The box represents the extraction of $r$ from $J / \psi$ electroproduction data [2] (see text).

observes a signal for a stronger antishadowing in the gluon case $(\approx 8 \%)$ than in the quark case $(\approx 1 \%)$. In the shadowing region, there is no evidence for a difference between quarks and gluons. It is, however, presently impossible to draw a firm conclusion in this region.

It turns out that the uncertainty in the right hand side of Eq. (10) comes predominantly from the error in $f_{2}$ so that, in Table 1, we report the uncertainty

$$
\delta r=\frac{\Delta f_{2}}{f_{3}-\frac{\alpha_{S}}{2 \pi} \frac{\Delta^{C}}{F_{2}^{C}}},
$$

where $\Delta f_{2}$ is the experimental statistical error on $f_{2}$. The systematic error is in the process of being assessed.

There are also theoretical approximations. Neglect of NLL corrections together with the use of Eq. (5) lead to an approximation presumably better than $20 \%$ for an absolute extraction of gluon density. Because the ratio $r(x)$ remains close to 1 in the whole range of $x$ being considered, they are hopefully even far better for the ratio we are evaluating.

The ratio $r$ has also been extracted from $J / \psi$ electroproduction [2] in the range $0.05 \leq$ $x \leq 0.15$, with the averaged result $\langle r\rangle=1.13 \pm 0.08$. In Fig. 2, we show this value as a rectangle the sides of which are the $x$ range of the data and the errorbar in $\langle r\rangle$. One can see that the two different extractions are in good agreement.

Using the result on $r$, one can estimate the consequence on $\Delta R=R^{\mathrm{Sn}}-R^{\mathrm{C}}$, the difference of the ratios of longitudinal to transverse virtual photon cross sections. In 
principle measurement of $\sigma_{L}$ at small $x$ provides a direct information on $G(x)$. This measurement is, however, difficult, therefore experimental analysis of $\sigma_{L}$ is still going on in the small $x$ range. Here we examine the problem by going in the opposite direction, that is to see whether the extracted ratio of gluon distributions, $r(x)$, is compatible with the NMC measurement of $\Delta R$ [1].

From the expression of $R$,

$$
R=\frac{F_{L}+\frac{4 M_{N}^{2} x^{2}}{Q^{2}} F_{2}}{F_{2}-F_{L}} \approx \frac{F_{L}}{F_{2}-F_{L}},
$$

assuming $\Delta R \ll R$ (see below), one finds

$$
\Delta R \approx R(1+R)\left(\frac{1}{f_{1}} \frac{F_{L}^{\mathrm{Sn}}}{F_{L}^{C}}-1\right)
$$

At next to leading order, $F_{L}$ is given by 9

$$
F_{L}(x)=\frac{\alpha_{S}}{2 \pi} x^{2} \int_{x}^{A} \frac{d y}{y^{3}}\left(\frac{8}{3} F_{2}(y)+2 \sum e_{i}^{2}(1-x / y) y G(y)\right)
$$

At small $x$, the integrals can be approximated in a way similar to the method leading to Eq. (5). One has [9]

$$
F_{L}(x) \approx \frac{\alpha_{S}}{2 \pi}\left(\frac{4}{3} F_{2}(2 x)+\frac{2 \sum e_{i}^{2}}{5.85} 2.5 x G(2.5 x)\right) .
$$

The $F_{2}$-terms are smaller than the $G$-terms, but they are not negligible in the whole $x$ range. A detailed analysis would require a better knowledge of absolute values of $F_{2}$ 's and $G$ 's, so we restrict ourselves to an estimate for $\Delta R$ by dropping the $F_{2}$-terms to simply end with

$$
\left.\frac{\Delta R}{R(1+R)}\right|_{x, Q^{2}} \approx \frac{r\left(2.5 x, Q^{2}\right)}{f_{1}\left(x, Q^{2}\right)}-1 .
$$

Simple parametrizations of $r$ and $f_{1}$ shows that this quantity varies very weakly with $x$ in the range $0.01 \leq x \leq 0.1$. It is about $0.08 \pm 0.01$. $R$ is about $0.1-0.3$ for the nucleon case in this $x$-range (and for the $Q^{2}$ of the data), so that one verifies the above statement that $\Delta R \ll R$, and one gets $0.01 \leq \Delta R \leq 0.03$. This is compatible with the new data from NMC [1]. We note, however, that the present precision of the data on $\Delta R$ unfortunatly does not give much constraint on the extracted ratio $r(x)$.

The present analysis can be compared with previous analysis [4, 5] of $J / \psi$-production in hadronic collisions [3]. In an older attempt [团, the experimental data have been all accounted for by gluon shadowing. The resulting ratio, $G^{\mathrm{Sn}} / G^{\mathrm{C}} \approx 1+(0.021 \pm$ $0.001) \ln x_{2} \ln 118 / 12$, would be much below our determined ratio in Fig. 2 for $x \geq 0.01$. Recently, the same data have been reanalysed [5] on the basis of a Fock state decomposition of the charmonium states with an essential contribution from the $\left|(c \bar{c})_{8} g\right\rangle$ component, 
supposed to give the interaction of the precharmonium state soon after formation. This new analysis can explain the FNAL data without shadowing of the gluon distribution at variance with our analysis.

The shape of $r(x)$ obtained in this paper has been anticipated in [13] on the basis of the fulfilment of the momentum sum-rule. In order to get a momentum fraction for the gluons $\approx 2 \%$ higher in the Calcium nucleus than in a free nucleon, shadowing in the gluon distribution at $x<0.05$ was predicted to be compensated by a noticeable antishadowing around $x=0.1$. We can infer that the general feature discussed in 13 are in qualitative agreement with the data. The sum-rule fraction for the shadowing+antishadowing region, $x<x_{0} \approx 0.25$, is

$$
\gamma_{G}=\frac{\int_{0}^{0.25} d x(r(x)-1) x G(x)}{\int_{0}^{1} d x x G(x)} \approx 2 \%,
$$

in tin to carbon. This value is close to the estimated enhancement of the gluon fraction in Ca to D. Let us note for completeness that the contribution from $x>0.25$ to the momentum fraction carried by gluons is not totally negligible, in spite of the small gluon density, $G(x)$, at large $x$. Typically, a mean depletion of $10 \%$ in the interval $0.25 \leq x \leq 0.5$ (EMC effect for gluons) diminishes $\gamma_{G}$ from $2 \%$ to $1 \%$.

The range of $x$ values accessible via the NMC $F_{2}$-measurements $0.01 \leq x \leq 0.2$ is sufficient to estimate the importance of shadowing for the minijet production rate at RHIC. One needs, however, much smaller $x$ information, down to $x \approx 10^{-4}$, for heavy ion collision at LHC. To cover this range, HERA with nuclear beams would be very helpful. The scaling violation analysis shown here may have difficulties to be used since the standard evolution of the $F_{2}$ structure function, even with NLL corrections included, may be modified by non-leading twist terms coming from the merging of partons" [8]. It is therefore important to extend the inclusive measurements of $F_{2}$ to jet, open charm and charmonium productions.

We gratefully acknowledge two members of NMC, Antje Brüll and Andreas Mücklich, for providing us the data and related informations. We have also benefitted from discussions with participants of the working group on nuclei at HERA.

\footnotetext{
${ }^{1}$ Let us mention that this non-linear effect rather than being a limitation to gluon density analysis is to be seen as one of the very challenging effects to be studied at HERA.
} 


\section{References}

[1] A. Mücklich, Ph.D. thesis, Universität Heidelberg, 1995; A. Mücklich, talk given at the Workshop on Deep Inelastic scattering and QCD, Paris, 1995.

[2] NM Collab., P. Amaudruz et al., Nucl. Phys. B 371 (1992) 553.

[3] D.M. Alde et al., Phys. Rev. Lett. 66 (1991) 133, Phys. Rev. Lett. 66 (1991) 2285.

[4] S. Gupta and H. Satz, Z. Phys. C 55 (1992) 391.

[5] D. Kharzeev and H. Satz, Charmonium composition and nuclear suppression, preprint CERN-TH/95-214r, 1995.

[6] J. Hüfner and B. Kopeliovich, The relative $J / \psi$ to $\psi^{\prime}$ suppression in proton-nucleus and nucleus-nucleus collisions, preprint MPI-H-511-1995, 1995.

[7] H.J. Pirner, M. Kaluza and A.G. Schneider-Neureither, Phys. Rev. C 48 (1993) 877.

[8] A.H. Mueller and J. Qiu, Nucl. Phys. B 268 (1986) 427; J. Qiu, Nucl. Phys. B 291 (1987) 746 .

[9] R.G. Roberts, The structure of the proton, Cambridge University Press, Cambridge, 1989.

[10] K. Prytz, Phys. Lett. B 311 (1993) 286, B 332 (1994) 393.

[11] NM Collab., M. Arneodo et al., Phys. Lett. B 309 (1993) 222.

[12] NM Collab., M. Arneodo et al., Nucl. Phys. B 441 (1995) 12.

[13] L.L. Frankfurt, M. Strikman and S. Liuti, Phys. Rev. Lett. 65 (1990) 1725. 